\title{
Reconstitution de l'expérience des liqueurs de Blaise Pascal
}

\section{Armand Le Noxaïc et Pierre Lauginie}

\section{(2) OpenEdition}

\section{Journals}

Édition électronique

URL : http://journals.openedition.org/ccibp/408

DOI : $10.4000 /$ ccibp.408

ISSN : 2493-7460

Éditeur

Centre international Blaise Pascal

\section{Édition imprimée}

Date de publication : 6 décembre 2010

Pagination : 48-55

ISBN : 978-2-84516-498-7

ISSN : 0249-6674

Référence électronique

Armand Le Noxaïc et Pierre Lauginie, « Reconstitution de l'expérience des liqueurs de Blaise Pascal », Courrier du Centre international Blaise Pascal [En ligne], 32 | 2010, mis en ligne le 26 novembre 2015, consulté le 20 avril 2019. URL : http://journals.openedition.org/ccibp/408; DOI : 10.4000/ccibp.408

Ce document a été généré automatiquement le 20 avril 2019.

Centre international Blaise Pascal 


\title{
Reconstitution de l'expérience des liqueurs de Blaise Pascal
}

\author{
Armand Le Noxaïc et Pierre Lauginie
}

1 Au tout début du XXe siècle, Félix Mathieu avait écorné l'image de Blaise Pascal en l'accusant d'avoir produit un faux pour s'attribuer les mérites de l'hypothèse des effets de la pression atmosphérique concernant l'expérience du Puy de Dôme ${ }^{1}$. Félix Mathieu avait d'autre part ${ }^{2}$ remis en cause le témoignage de Roberval sur l'expérience des liqueurs faite par Pascal à Rouen, pour laquelle il aurait utilisé des tubes longs de 46 pieds $^{3}$ (environ $15 \mathrm{~m}$ ) remplis d'eau et de vin. Selon lui, Pascal n'aurait utilisé qu'un seul tube contenant du vin: «Pascal fit, dans la cour de la verrerie, avec un grand tube rempli de vin, une expérience qui n'ajoutait rien à ce qu'avait dit Galilée ${ }^{4}$ ». À la lecture des trois articles ${ }^{5}$ offensifs de Félix Mathieu parus dans la Revue de Paris, Blaise Pascal apparait comme un opportuniste sans scrupules dont l'originalité des expériences scientifiques serait contestable.

2 Concernant l'expérience des liqueurs à Rouen, un pas de plus avait été franchi par Alexandre Koyré dans les années 1950, lorsqu'il avait laissé planer le doute sur sa réalisation par Pascal ${ }^{6}$. Un phénomène de bouillonnement de l'eau (et du vin) aurait dû être relaté par Pascal, comme on le constata en 1950 au Palais de la Découverte lorsque l'expérience y fut reproduite avec un long tube de verre et de l'eau. Il avança comme autre argument la très grande difficulté à se procurer un tube de verre long de 15 mètres. Alexandre Koyré accusait Pascal de ne pas avoir dit toute la vérité sur cette expérience, en laissant sous-entendre qu'il pouvait même ne jamais l'avoir réalisée.

3 En 1985, Kimiyo Koyanagi, au Japon, avait fait une reconstitution de l'expérience utilisant de l'eau et du vin, et avait constaté que le vin montait moins haut que l'eau ${ }^{7}$, en désaccord avec ce que relatait Roberval. Elle défend depuis sa position qui est de dire que Pascal n'a pas fait cette expérience des liqueurs, ni avec de l'eau, ni avec du vin ${ }^{8}$.

Ce réquisitoire en crescendo contre Pascal mérite que l'on s'y intéresse, car il fragilise l'image d'un Pascal expérimentateur honnête et rigoureux. Parmi ces attaques, il est nécessaire de discerner celles qui concernent davantage Roberval. Nulle part Pascal ne dit avoir conjointement fait l'expérience avec l'eau et le vin. Dans les Expériences nouvelles 
touchant le vide 9 Pascal donne les hauteurs de 32 pieds pour le vin et 31 pieds pour l'eau, sans indiquer s'il les a mesurées simultanément. On ne peut pas accuser Pascal de ne pas avoir fait ce dont il ne parle pas. Une reconstitution de l'expérience des liqueurs avec de l'eau et du vin où le vin monterait plus haut que l'eau sans constater de phénomène de bouillonnement des liquides rendrait caduques toutes ces critiques, qu'elles concernent Pascal ou Roberval.

5 Le 11 février 2010, une reconstitution de l'expérience des liqueurs fut réalisée à l'Institut Universitaire de Technologie d'Orsay ${ }^{10}$. Le vin s'est positionné à environ $18 \mathrm{~cm}$ au dessus de l'eau, lorsque les extrémités inférieures des tuyaux contenant les liquides ont été débouchées, sans qu'aucun phénomène de bouillonnement ne soit visible depuis le bas du dispositif expérimental. Au fil du temps, l'écart entre les hauteurs du vin et de l'eau a augmenté, tendant vers une trentaine de centimètres - en accord avec le «pied» de différence indiqué par Pascal dans les Expériences nouvelles touchant le vide. La réussite de cette reconstitution repose intégralement sur l'interprétation des informations données par Roberval dans sa Première narration sur le vide, comme nous allons le voir.

\section{Les tubes utilisés par Pascal et pour la reconstitution de son expérience}

6 Commençons par souligner la fragilité de l'argument avancé par Alexandre Koyré sur l'impossibilité de faire des tubes longs de 15 mètres au XVII siècle. Des tubes plus courts ont pu être soudés par les verriers de Rouen pour parvenir à un tube de la longueur désirée par Pascal ${ }^{11}$. Kimiyo Koyanagi est d'accord sur ce point: "Il est vrai que l'argumentation de Koyré contient quelques points faibles. Par exemple, il fait remarquer la difficulté qu'on a éprouvée en 1950 lors de la fabrication d'un tuyau de verre de 15 mètres au Palais de la Découverte. Mais bien sûr, fabriquer un grand tuyau en joignant des tuyaux de petite taille ne change point l'effet de l'expérience ${ }^{12}$ ».

7 Pour la reconstitution d'Orsay ont été utilisés des tubes ${ }^{13}$ souples en PVC transparents de diamètre intérieur $12 \mathrm{~mm}$ et de longueur 12 mètres. Ces tubes ont été fixés sur des barres en aluminium à l'aide d'attaches, afin d'éviter qu'ils ne s'allongent une fois placés à la verticale.

\section{Les conditions climatiques de l'expérience de Pascal et de sa reconstitution}

Selon Roberval, Pascal fit à plusieurs reprises l'expérience dans la cour de la verrerie de Rouen durant les mois de janvier et/ou février $1647^{14}$. L'expérience aurait donc été faite à l'extérieur et dans des conditions de température hivernale. Or les propriétés des liquides évoluent en fonction de la température, notamment leurs pressions de vapeur saturante ${ }^{15}$ . Nous savons aujourd'hui qu'au-dessus des colonnes d'eau et de vin se trouvent entre autres des vapeurs de ces liquides - mais Pascal ignorait évidemment le phénomène de pression de vapeur saturante ${ }^{16}$. Ces pressions de vapeur saturante dépendent fortement de la température. La loi de Clapeyron ${ }^{17}$ nous permet de prévoir la valeur de ces pressions pour une température donnée. En janvier ou février 1647, la température des liquides devait être inférieure à $10^{\circ}$ Celsius comme ce fut le cas lors de notre reconstitution en février 2010. Pour se rapprocher davantage des conditions expérimentales de l'époque, il 
était impératif de faire cette expérience à l'extérieur et durant l'hiver, en contrôlant la température des liquides. Ces vapeurs se situent au-dessus des liquides et leurs hauteurs en dépendent, la pression de vapeur s'opposant à la pression atmosphérique. Plus la pression de vapeur d'un liquide sera élevée moins il montera haut dans le tube le contenant. Faire cette expérience durant l'été ou dans un local chauffé éloignerait trop des conditions requises pour faire une reconstitution. Le tableau suivant permettra de se rendre compte des effets de la température sur les pressions de vapeur saturante de l'eau, du vin à $11 \%$ d'alcool, et d'un vin muté ${ }^{18}$ à $20.5 \%$ d'alcool $^{19}$. Lorsque la température des liquides diminue, la différence entre les pressions de vapeur saturante du vin et de l'eau diminue. D'une manière générale - quelque soit la température - la pression de vapeur saturante du vin plus élevée que celle de l'eau incite le vin à monter moins haut que l'eau, mais ce phénomène s'amoindrit lorsque la température diminue. Lors de la reconstitution d'Orsay, la température des liquides était de $7^{\circ}$ Celsius et la pression atmosphérique de 745.5 torr pour une altitude de $165 \mathrm{~m}$ par rapport au niveau de la mer.

Les valeurs de Pvap.sat. sont indiquées en torr dans le tableau.

\begin{tabular}{|l|l|l|l|l|l|}
\hline $\begin{array}{l}\text { Température du } \\
\text { liquide } \\
\text { Celsius }\end{array}$ & $\begin{array}{l}\text { Pvap.sat. de } \\
\text { l'eau } \\
\text { (colonne A) }\end{array}$ & $\begin{array}{l}\text { Pvap.sat. du } \\
\text { vin à 11\% } \\
\text { (colonne B) }\end{array}$ & $\begin{array}{l}\text { Pvap.sat. du à 20.5\% } \\
\text { vin (colonne C) }\end{array}$ & $\begin{array}{l}\text { (colonne B) (colonne } \\
\text { A) }\end{array}$ & $\begin{array}{l}\text { (colonne } \\
\text { (colonne A) }\end{array}$ \\
\hline 5 & 6.8 & 7.9 & 8.9 & 1.1 & 2.1 \\
\hline 10 & 9.4 & 11 & 12.3 & 1.6 & 2.9 \\
\hline 20 & 17.4 & 20.3 & 22.9 & 2.9 & 5.5 \\
\hline 25 & 23.3 & 27.3 & 30.7 & 4 & 7.4 \\
\hline 30 & 31 & 36.2 & 40.7 & 5.2 & 9.7 \\
\hline
\end{tabular}

Remarque : Une pression de 1 Torr correspond environ à 1,4 cm d'eau ou de vin.

\section{La densité du vin utilisé par Pascal et pour la reconstitution}

Toujours selon Roberval, Pascal aurait calculé - avant de faire l'expérience - les hauteurs d'eau et de vin: «Chez lui, le très habile $M$. de Paschal avait fait le calcul des poids de l'eau et du vin par rapport à celui du vif-argent, pour en déduire la hauteur convenable à chacun d'eux pour que, à ces hauteurs respectives, ils eussent le même poids; et il avait trouvé, étant admise la hauteur susdite du vif-argent, soit 2 pieds $7 / 24$, qu'il fallait environ 31 pieds $1 / 9$ d'eau et à peu près 31 pieds $2 / 3$ de vin ${ }^{20} »$. Cette information est de la première importance pour notre reconstitution, car elle permet de calculer la densité du vin utilisé par Pascal. Comme nous allons le voir, ses valeurs de hauteur sont le fruit de calculs très précis faits par Pascal. En effet, si on divise la hauteur d'eau indiquée cidessus par celle de mercure, on trouve une valeur comprise entre 13.57 et 13.58 - soit la valeur de la densité du mercure avec une excellente précision ${ }^{21}$. Si on divise maintenant la hauteur d'eau par celle de vin, on trouve une densité du vin égale à 0.982 . Or même 
aujourd'hui - à une époque où les vins sont davantage filtrés - on ne rencontre jamais un vin ayant une densité aussi faible. La densité d'un vin est comprise entre 0.990 et 0.994 , en fonction de son degré d'alcool et de sa température. Pourquoi Pascal considère-t-il une densité bien plus faible? Une erreur est peu envisageable, pourquoi se tromperait-il sur la densité du vin et non sur celle du mercure, qui plus est lors d'un calcul élémentaire ? La solution la plus plausible ${ }^{22}$ est que Pascal a fait des calculs pour un vin muté, c'est-à-dire un vin enrichi en alcool. Une concentration plus élevée en éthanol diminue la densité du vin car la densité de l'éthanol est plus faible que celle de l'eau contenue également dans le $v^{2}{ }^{23}$. Pascal a-t-il volontairement enrichi le vin dont il disposait en alcool, ou celui-ci l'était-il déjà ? Nous discuterons de cela au paragraphe suivant. Pour la reconstitution d'Orsay, nous avons constitué un "vin » dont la densité est égale à 0.982. Pour cela, nous avons fait un mélange de vin rouge (marque «La Villageoise » degré d'alcool 11\%) et d'armagnac (marque « Reflets de France », Bas-Armagnac 12 ans d'âge, degré d'alcool $40 \%$ ). En mélangeant 1 volume d'armagnac pour 2 volumes de vin, nous sommes parvenus à une densité du mélange égale à 0.982 mesurée à l'aide d'un aréomètre. Le degré d'alcool du mélange s'élève environ à $20.5 \%$. Le choix de l'armagnac ${ }^{24}$ plutôt qu'une autre eau de vie n'est pas obligatoire, l'important est que la densité du «vin» confectionné soit la même que celle du vin utilisé par Pascal.

\section{Quelques autres paramètres importants pour la reconstitution}

10 Le conditionnement du vin et celui de l'eau ont également leur importance. À l'époque de Pascal, le vin était contenu en tonneau. La bouteille de vin en verre venait d'être inventée par le diplomate anglais Kenelm Digby dans les années 1630, mais n'était certainement pas généralisée. Le problème de la conservation du vin se posait. L'ajout d'eau de vie au vin permet d'améliorer sa conservation, car cela empêche la fermentation du vin de se poursuivre. L'adjonction d'eau de vie est ancienne ${ }^{25}$; elle fut décrite ${ }^{26}$ par Arnaud de Villeneuve dès le XIII ${ }^{e}$ siècle dans son traité Liber de vinis. Il est envisageable que le vin utilisé par Pascal ait été enrichi en alcool à ces fins ou pour des raisons gustatives. Prendre du vin d'aujourd'hui contenu en bouteille pour faire une reconstitution nous éloignerait trop de la réalité pour encore une autre raison : le vin renferme du dioxyde de carbone dissous provenant de la fermentation qui a permis de transformer les sucres du jus de raisin en alcool. Il est fort possible que ce taux de dioxyde de carbone dissous ne soit pas le même en fonction de son conditionnement. Un vin aéré contiendra moins de dioxyde de carbone qu'un vin mis en bouteille dès son élaboration. Pour notre reconstitution, nous avons laissé le "vin » confectionné s'aérer. L'eau utilisée n'échappe pas à ces précautions. Blaise Pascal a certainement utilisé de l'eau de source ou de rivière, qui ne renfermait pas la même quantité de gaz dissous que l'eau dont nous disposons au robinet. Pour notre reconstitution, nous avons aéré l'eau utilisée comme nous l'avons fait pour le vin. Si le vin renferme trop de dioxyde de carbone, il se met à mousser lorsqu'il descend dans le tube - ce que nous avons constaté lors d'une première tentative faite en décembre 2009. Ces précautions permettent également de considérablement minimiser le phénomène de bouillonnement des liquides - qui avait été constaté au Palais de la Découverte dans les années 1950. Jean Mesnard commentant les critiques d'Alexandre Koyré fit cette remarque: "Est-il impossible que Pascal ait pu trouver le moyen d'empêcher le phénomène du bouillonnement? N'est-ce pas une illusion 
fréquente chez l'homme d'aujourd'hui que de trouver quasi impossibles des entreprises que l'homme d'autrefois a effectivement réalisées? $»^{27}$. La réponse est oui. On peut encore davantage dégazer les liquides en les aérant mécaniquement puis en les chauffant ${ }^{28}$. Blaise Pascal a très bien pu le faire. Il a dû - comme nous l'avons fait - remplir avec précaution les tubes pour ne pas y enfermer des bulles d'air. Et, si cela s'est produit, il a dû alors patiemment les chasser - tout cela est possible, puisque nous l'avons fait lors de notre reconstitution. Malgré toutes les précautions possibles, il restera toujours de l'air et autres gaz dissous - mais en bien moindre quantité. Ces gaz dissous remonteront les tubes débouchés placés à la verticale, sous la forme de bulles indiscernables lorsqu'elles se situent au bas des tubes, mais grossissant progressivement lors de leur ascension. Une partie des gaz dissous se retrouvera dans les espaces apparemment vides créés au-dessus des liquides. La question est de savoir maintenant si Pascal a pu voir ces bulles dont l'importance a pu être néanmoins fortement minimisée par les différentes précautions énumérées? 
Le dispositif a été dressé à la verticale. Un escalier permettra de visualiser le comportement des fluides lors de l'expérience. 


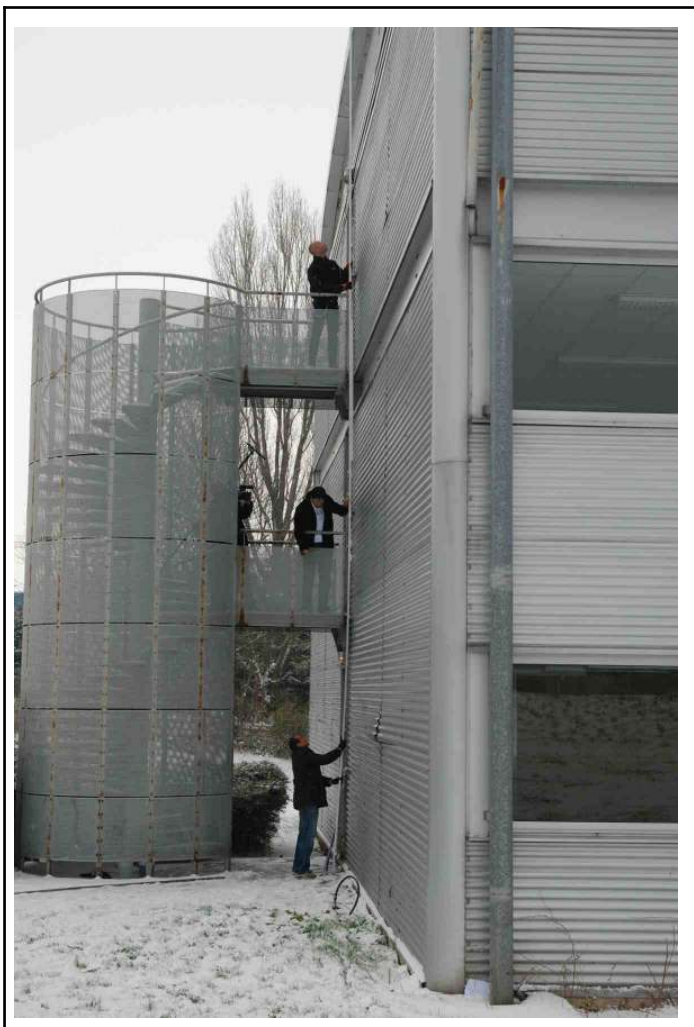

Les extrémités inférieures des tubes sont plongées dans des seaux contenant de l'eau à une température hivernale.

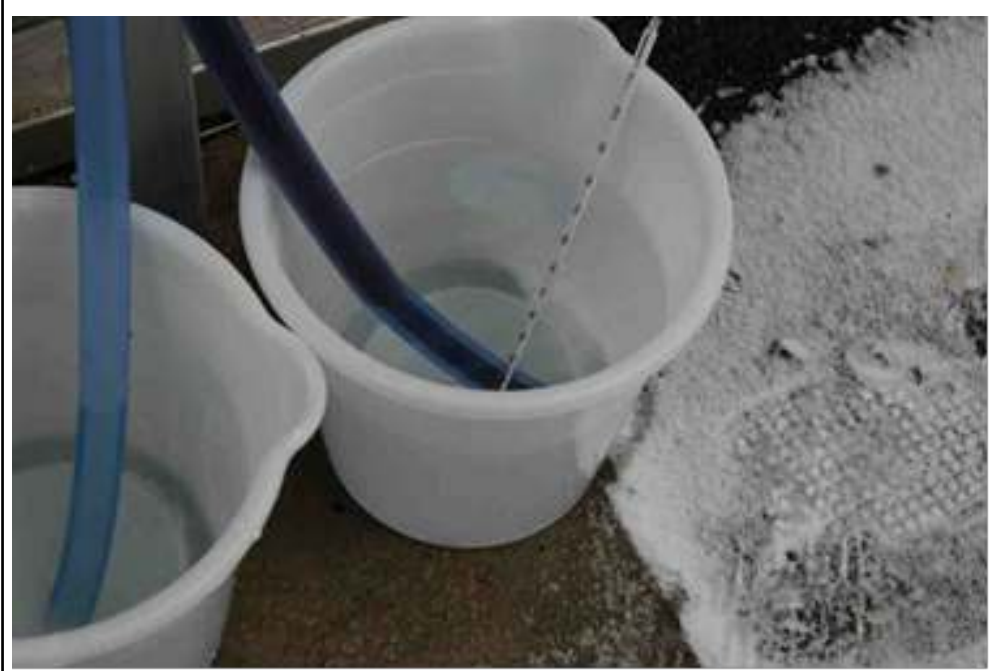

Après avoir débouché les extrémités inférieures des tubes, les liquides descendent et se stabilisent pour équilibrer la pression atmosphérique. Le vin m

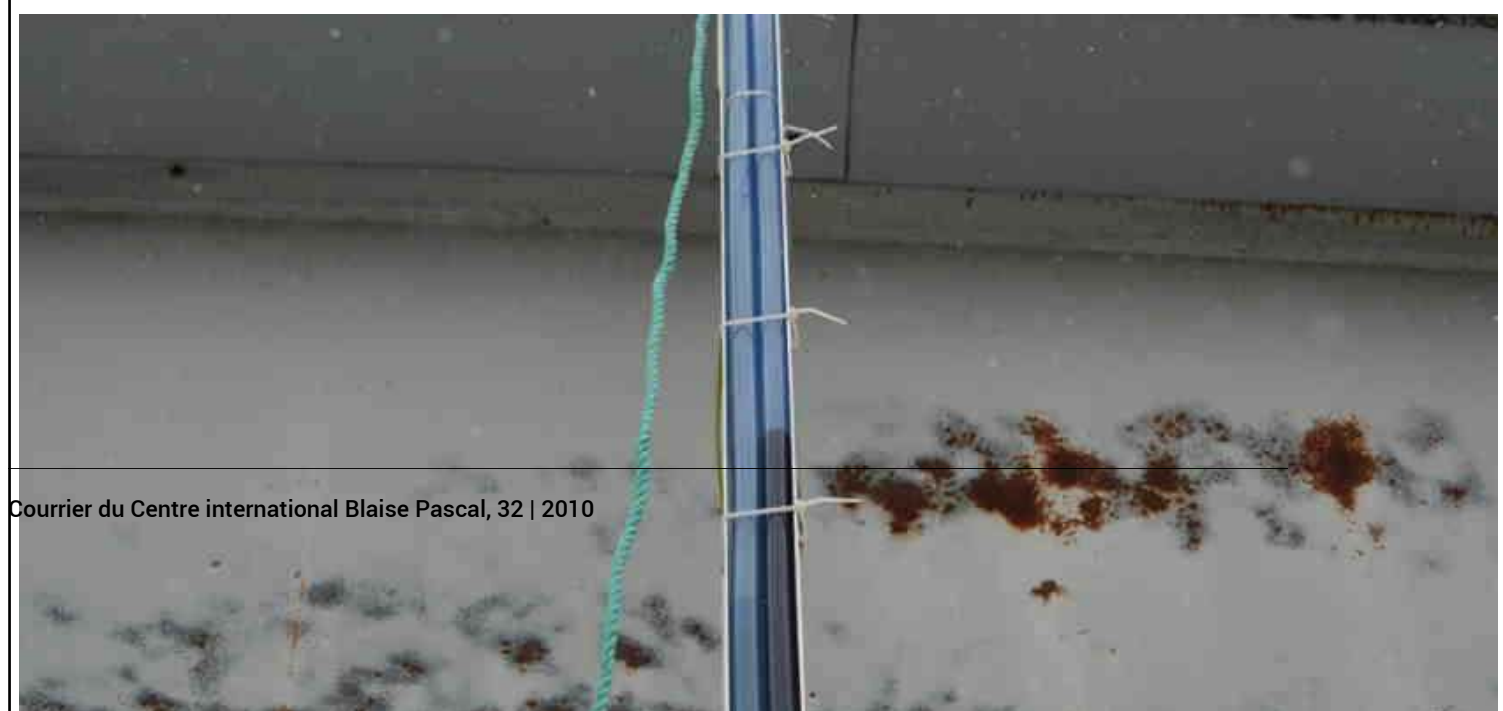




\section{Où se situait Pascal lors de l'expérience ?}

11 Nous ne savons pas s'il y avait dans la cour de la verrerie de Rouen un bâtiment suffisamment haut permettant de voir sans difficultés le comportement des fluides en haut des tubes ${ }^{29}$. Si ce bâtiment existait, les tubes ont très bien pu être adossés à un de ses murs. On peut imaginer que la présence d'une fenêtre située à une dizaine de mètres de hauteur aurait permis à Blaise Pascal d'observer les niveaux finaux des liquides contenus dans les tubes. D'autres fenêtres auraient pu permettre de visualiser ce qui se passait dans ces tubes pour des hauteurs moindres. Mais rien ne prouve l'existence d'un tel bâtiment en ce lieu. Les éléments que l'on dispose sont ceux rapportés par Roberval qui déclare que les tubes étaient « attachés à un mât de navire, si bien équilibré par des machines conçues pour cela qu'on pouvait facilement le dresser et l'abaisser selon le besoin ${ }^{30} »$. Aucune référence n'est faite à un quelconque bâtiment facilitant l'expérience. Comment Blaise Pascal a-t-il pu alors discerner les niveaux des liquides situés à une dizaine de mètres de hauteur?

Lors de notre reconstitution, les spectateurs présents situés au pied de notre dispositif ont pu les discerner à l'œil nu. Blaise Pascal était très probablement situé au pied de son dispositif, muni éventuellement d'une longue-vue. Pour notre reconstitution, nous avions une lunette de grossissement $\mathrm{x} 5$. Des longues-vues d'un tel grossissement existaient à l'époque de Pascal. Celle-ci nous a permis de mieux voir du bas les niveaux supérieurs de l'eau et du vin, mais elle ne permettait pas de voir les quelques bulles qui éclataient en haut des tubes. Pour répondre à Alexandre Koyré, Blaise Pascal a très bien pu ne pas parler du bouillonnement des liquides pour la simple raison qu'il ne pouvait le voir - même muni d'une longue-vue. Lors de notre reconstitution, nous avons pu remarquer que jusqu'à six mètres de hauteur, aucune bulle n'était discernable. Pour voir la faible ébullition se produisant en haut des liquides, il était nécessaire de se trouver à leur hauteur - ce que nous pouvions faire grâce à la présence d'un escalier extérieur contre lequel nous avions fixé notre dispositif.

Cependant, la seconde narration de Roberval sur le vide laisserait penser que des bulles d'air aient été visualisées lors de l'expérience de Pascal: "de petites gouttes d'air montent de la partie inférieure du tube, si petites qu'elles égalent à peine le plus petit grain de mil : lesquelles, au fur et à mesure de la montée, grandissent de plus en plus ; jusqu'à ce qu'elles parviennent au sommet de la liqueur contenue dans le tube, où on les voit si grandes qu'elles occupent facilement toute la largeur du tube ${ }^{31}$ ». La rédaction de cette seconde narration aurait été entreprise par Roberval en mai $1648^{32}$ - à une époque où l'auteur avait changé d'avis concernant la réalité du vide. L'expérience de la vessie de carpe inventée par Roberval en mars 1648 avait fait basculer ses convictions. Une vessie de carpe vidée et nouée avait été placée à l'intérieur dans le haut d'un tube de Torricelli, celle-ci s'enflait alors immédiatement. L'air résiduel avait donc la capacité de se dilater pour empêcher le vide. Roberval rejoint alors le camp de ceux qui soutenaient que l'espace vide au-dessus des colonnes de liquides était en réalité de l'air raréfié. Pourquoi Roberval n'a-t-il pas évoqué ces bulles d'air dans sa première narration? Il est envisageable qu'il ait extrapolé ce qu'il constatait pour des tubes contenant du mercure à ceux contenant du vin ou de l'eau. Mais, il a pu également passer sous silence ce phénomène lors de sa première narration. Ces deux hypothèses ne constituent pas 
néanmoins des arguments contre la réalité de la réalisation par Pascal de l'expérience des liqueurs.

En conclusion, nous soulignerons le fait que si nous avons réussi avec nos modestes moyens à retrouver les résultats de Blaise Pascal, il n'y a aucune raison pour que celui-ci n'ait pas fait cette expérience. A Rouen, tous les éléments étaient réunis pour que Pascal puisse entreprendre cette expérience : la meilleure verrerie du Royaume, les moyens financiers apportés par son père, la main d'œuvre locale. La réalité d'une expérience aussi spectaculaire était de plus aisément vérifiable, or il n'existe aucun texte de l'époque la mettant en doute. L'insuccès de nos prédécesseurs à retrouver ce qu'a constaté et montré Pascal est inhérent à la difficulté de faire une reconstitution d'une expérience scientifique passée. Chaque expérience est unique. Une reconstitution ne peut que prétendre s'en rapprocher étant donnés le grand nombre de paramètres physiques qui y interviennent. Cependant certains paramètres jouent souvent un rôle plus important que d'autres. Pour cette expérience, la densité du vin ainsi que la température des liquides et la quantité de gaz dissous dans les liquides sont trois paramètres primordiaux. Grâce à la narration de Roberval nous avons pu disposer de ces éléments en interprétant certains de ses passages. Sans l'existence de cette narration, il aurait été bien difficile juste à la lecture des Expériences nouvelles touchant le vide de réussir cette reconstitution. Pascal dit qu'il utilise $\mathrm{du}$ « vin bien rouge ${ }^{33}$ » mais ne donne pas davantage de détails sur les caractéristiques de ce vin. Les calculs de Pascal communiqués par Roberval ont permis de mettre en évidence le fait surprenant que le vin utilisé était bien plus léger que d'ordinaire. Si Pascal avait volontairement, et sans le dire, enrichi le vin en alcool pour que sa hauteur soit plus grande que celle de l'eau, il aurait su pertinemment que personne n'aurait pu constater la même chose que lui. Notre hypothèse est que le vin utilisé par Pascal était un vin muté, déjà enrichi en alcool.

\section{NOTES}

1. F. MATHIEU, Revue de Paris, $1^{\mathrm{er}}$ mars 1907, pp. 179-206.

2. F. MATHIEU, Revue de Paris, $1^{\mathrm{er}}$ avril 1906, pp. 565-589.

3. Un pied de l'époque correspond environ à $32,5 \mathrm{~cm}$.

4. F. MATHIEU, Revue de Paris, $1^{\mathrm{er}}$ avril 1906, p. 580.

5. F. MATHIEU, Revue de Paris, $1^{\mathrm{er}}$ avril 1906, 15 avril 1906, $1^{\mathrm{er}}$ mars 1907.

6. A. KOYRE, Pascal, l'homme et l'œuvre, Cahiers de Royaumont, Philosophie $n^{\circ} 1$, Éd. de Minuit, 1956, pp. 273-278.

7. En août 2008, des chercheurs hongrois ont constaté la même chose lors d'une reconstitution. Voir S.L. ATTILA, S. ISTVAN, S. CSABA, «A Torricelli - kiserlet », Fisikai Szemle, 2009/1.

8. K. KOYANAGI, «Cet effrayant petit livret... Expériences nouvelles touchant le vide de Blaise Pascal », Les Pascal à Rouen 1640-1648, Publications de l'Université de Rouen, 2001, pp. 137-157.

9. J. MESNARD, CEuvres Complètes de Pascal, Tome 2, Desclée de Brouwer, (quatre tomes parus, 1964-1992), pp. 493-508.

10. De nombreuses personnes ont participé d'une manière ou d'une autre à cette reconstitution. Nous remercions particulièrement nos collègues Laurent Nagat, Alain Angel, Jean Claude Serres, 
et Fabien Legrand; ainsi que les étudiants qui ont fait leur projet tuteuré sur ce sujet : Leentie Edwiges, Doriane Guilleux, Caroline Grange, Thibault Oliveira, Thibault Payan, et Nicolas Tissot.

11. J. MESNARD, op. cit.3, p. 462 et p. 469.

12. C. MAZAURIC, « Note sur la verrerie de Saint-Sever au temps d'Étienne Pascal », Les Pascal à Rouen 1640-1648, Publications de l'Université de Rouen, 2001, pp. 159-178.

Voir p. 177: «Mais notre recherche patiente nous conduit à penser que l'argument de la prétendue incapacité technique des verriers de Rouen au XVII ${ }^{\mathrm{e}}$ siècle, à produire ce qui parut nécessaire aux Pascal et à Pierre Petit pour produire à Rouen des variantes fondamentales des expériences d'Italie, n'a aucune validité ».

13. K. KOYANAGI, op. cit., p. 141.

Masao Uchida avait également exprimé cette idée: « Since making a glass tube 2 to 3 meters long by hand is not a difficult task, it might have been possible to make several such tubes and connect them with lute or melt and connect both ends of the tubes".

Voir M. UCHIDA, Bulletin of the Faculty of Humanities, Wako University, n 17, 1982.

14. Tubes commercialisés par l'entreprise Hozelock sous le nom de «tublait ». Le tublait a une paroi dont l'épaisseur est de $5 \mathrm{~mm}$, ce qui lui permet d'être utilisé comme tube à vide.

15. J. MESNARD, op. cit. , p. 462 et p. 469.

16. Cette pression de vapeur est dite « saturante ", car si la pression au-dessus d'un liquide lui est inférieure, il se vaporise jusqu'à atteindre sa pression de vapeur saturante. Lorsque l'eau et le vin descendent dans les tubes, il y a une dépression en haut des tubes, ce qui provoque une vaporisation partielle des liquides jusqu'à ce que leurs pressions de vapeur saturante soient atteintes.

17. A. LE NOXAÏC, « Le vide mis en évidence par Pascal est-il exempt d'atomes ? », L'atomisme aux $\mathrm{XVII}^{e}$ et XVIII ${ }^{e}$ siècles, textes réunis et édités par Jean Salem, Publications de la Sorbonne, 1999.

18. (avec Pvap.sat. en torr et $\mathrm{T}$ en Kelvin) :

Loi de Clapeyron pour l'eau : Ln Pvap.sat $=20.33-5120 / \mathrm{T}$.

Loi de Clapeyron pour l'éthanol : Ln Pvap.sat = 19.16 - 3956.07/(T-35.62).

19. Nous considérons ici le cas d'un vin enrichi en alcool, ce qui sera expliqué au chapitre suivant traitant de la densité du vin utilisé par Pascal.

20. J. MESNARD, op. cit., t. 2, p. 469.

21. Entre 15 et $30^{\circ} \mathrm{C}$, la densité du mercure est comprise entre 13,57 et 13,58.

22. En filtrant le vin pour éliminer une partie des résidus secs qu'il contient, il est également possible de diminuer sa densité. Cependant un tel filtrage - en supposant qu'il soit possible aurait eu pour effet d'éliminer une partie des colorants naturels du vin or Pascal dit utiliser un « vin bien rouge ». De plus, Pascal ne signale pas avoir fait une telle manipulation.

23. Le vin est constitué notamment d'eau, d'éthanol et de résidus secs (17 à 30 grammes/litre). À $20^{\circ}$ Celsius, la densité de l'éthanol est égale à 0.791, tandis que celle de l'eau est de 0.998 .

24. Le choix de l'armagnac découle de la préférence d'utiliser une eau de vie de vin qui existait déjà à l'époque de Pascal.

25. J.C. MARTIN, Les hommes de science, la vigne et le vin de l'Antiquité au XIXe siècle, Éditions Feret, 2009.

26. Nous remercions Madame Véronique Lemoine, conseillère scientifique et culturel au Centre Culturel du Vin de Bordeaux, de nous avoir fourni ces informations concernant l'histoire du vin.

27. J. MESNARD, op. cit., t. 2, p. 494.

28. Le vin doit être chauffé à une température raisonnable pour que l'alcool ne s'évapore pas. Il est nécessaire de ne pas chauffer au-delà de $60^{\circ} \mathrm{C}$. Le vin contient de l'éthanol qui s'évapore audessus de $78.5^{\circ} \mathrm{C}$, et du méthanol (en plus faible quantité) qui s'évapore au-dessus de $64.5^{\circ} \mathrm{C}$.

29. C. MAZAURIC, op.cit. , p. 160 : «Nous ne disposons ni des tubes, ni des mâts, pas même de leurs dessins, ni du plan exact du lieu précis ou des bâtiments où se seraient produites les expériences de Rouen ». 
30. J. MESNARD, op. cit., p. 462.

31. J.MESNARD, op. cit., pp. 608-609.

32. Ibid., p. 603.

33. Ibid., p. 502.

INDEX

Mots-clés : Pascal, expérience, liqueur, pression atmosphérique, physique, vide

Keywords : Pascal, expérience, liquor, atmospheric pressure, physics, vacuum

\section{AUTEURS}

\section{ARMAND LE NOXAÏC}

Université Paris-Sud, Laboratoire Étude sur les Sciences et les Techniques (EST) Groupe d'Histoire et Diffusion des Sciences d'Orsay (GHDSO), Orsay, F-91405.

Armand.le-noxaic@u-psud.fr

\section{PIERRE LAUGINIE}

Université Paris-Sud, Laboratoire Étude sur les Sciences et les Techniques (EST) Groupe d'Histoire et Diffusion des Sciences d'Orsay (GHDSO), Orsay, F-91405.

Pierre.lauginie@u-psud.fr 